\title{
Ecological Security Evaluation for Marine Ranching Based on the PLTS/ANP Method: A Case Study of Rongcheng
}

\author{
Yuan-Wei Du $(\mathbb{D}$ and Qiong Song (iD \\ Management College, Ocean University of China, Qingdao 266100, China \\ Correspondence should be addressed to Yuan-Wei Du; duyuanwei@ouc.edu.cn
}

Received 8 June 2021; Revised 15 July 2021; Accepted 4 January 2022; Published 21 January 2022

Academic Editor: Hassan Zargarzadeh

Copyright (C) 2022 Yuan-Wei Du and Qiong Song. This is an open access article distributed under the Creative Commons Attribution License, which permits unrestricted use, distribution, and reproduction in any medium, provided the original work is properly cited.

\begin{abstract}
The evaluation index system of ecological security of marine ranching (MRES) is based on the assumption that there is independence among evaluation indexes in the existing studies, which ignores the complex interactive paths of marine ranching as an artificial ecosystem. In this study, the MRES evaluation network model that includes interdependent relationships is established based on the Driver-Pressure-State-Impact-Response model and the analytic network process method. Then, the probabilistic linguistic term sets and analytic network process methods are used to calculate the weights of the evaluation indexes of MRES. The overall evaluation value and the contribution rate of clusters are consequently defined and analyzed to reflect the performance of MRES. Finally, a case study is carried out for evaluating the MRES of marine ranches in Rongcheng by means of the proposed method. The conclusions are summarized as follows: (1) The weights of clusters are ranked as Responses $>$ Impact $>$ Driver $>$ State $>$ Pressure, and "scientific management of fishery resources" is the most important index; (2) the MRES performance of marine ranches in the city of Rongcheng is at the medium security grade on the whole, and all 11 samples are driven by the response.
\end{abstract}

\section{Introduction}

Marine ranching is an ecological system based on the principle of ecology. It has the goals of environmental protection, resource conservation, and sustainable fishery output [1]. It follows natural productivity and uses modern engineering technology and management models to construct habitat restoration and artificial multiplication of marine resources. Research on marine ranching started later in China than elsewhere. The concept of marine ranching was first proposed by Zeng in 1979 [2]. After years of development, China's marine ranching has made great achievements in the number of ranches, construction scale, technical level, output, development mechanism, and other parameters [3]. Marine ranching is a major agricultural development strategy in China at present. It is a new fishery mode and marine economy that pursues the growth and protection of fishery resources along with the improvement of the marine ecological environment. It has ecological, economic, and social benefits [4]. In China, current research on marine ranching mainly focuses on technology, output, mechanism, and other aspects of the establishment and management of the ranching [1]. However, in terms of the ecosystem, although some studies have been conducted, such as the research on the structure and function of the food web for ecological safety, the research is still in the developing stage [5].

With the increasing ecological problems in the construction of marine ranching in China, it is of practical significance to study the ecological security of marine ranching (MRES) [6]. MRES refers to the overall balance of the resource structure and marine environment of the artificial ecosystem of marine ranching to achieve the objectives of environmental protection, resource conservation, and sustainable fishery output [7]. MRES is an important means to ensure the enhancement of fishery resources and the improvement of the ecological environment. Yang et al. [1] pointed out that it is one of the technological means for 
future marine ranching to develop a monitoring platform for the ecological security and environmental protection of marine ranching; the authors also suggested using multiple models to predict and evaluate the safety of marine ranching. $\mathrm{Du}$ and Sun [6] researched the influence paths of MRES in China and reported useful findings for managing MRES. Du and Gao [8] evaluated the ecological effects of marine ranching by comprehensively considering social and economic factors and made a systematic evaluation of MRES to provide theoretical guidance for the management practices of marine ranching. Qin et al. [9] analyzed the influencing factors of spatial variation of national marine ranching in China. Wan et al. [10] evaluated the sustainability of the supply chain of natural marine ranching and applied their novel model to determine marine ranching's sustainable performance.

In the existing literature, the research on MRES has achieved phased results, such as clarifying the concept of MRES, trying to build the evaluation index of MRES, proposing the evaluation method of MRES, and discussing the influence paths of MRES. These studies' results have important reference values for follow-up studies [11, 12]. However, the evaluation of MRES is based on the basic assumption that the indexes are independent of each other. For example, the analytic hierarchy process (AHP), which has been applied in many research about decision-making $[13,14]$, is a method that requires the independent relationship between elements/indexes, and it requires that the structure model is hierarchical and linear [8]. In other words, the research results of AHP will be invalid if there are interaction relationships between the indexes. The study in "influence paths of MRES" [6] indicated that marine ranching is an artificial ecosystem, and there are influence paths among its internal indexes. This means that it is questionable to simply use AHP to evaluate MRES. Based on this, how to make a more scientific evaluation of MRES on the basis of fully considering the influence relationships of indexes is an important issue to be studied. Considering the above information, this study chooses the analytic network process (ANP) to evaluate MRES.

ANP is an extended and complementary form of AHP, which was proposed and developed by Saaty [15-17]. ANP makes up for the limitations of the AHP method, which include being applicable to only linear structure $[17,18]$, and releases the restriction of hierarchical structure requirement because this method provides a framework that considers the interrelationships within a cluster and among different clusters between all evaluation metrics (criteria) [15]. The ANP method constructs a network system instead of the tree-shaped hierarchical structure of the AHP, thus overcoming the problem of dependence and feedback among criteria or alternatives [19]. ANP has been widely applied in various fields and has proven to be effective in solving decision-making problems, such as supply chain management [20], risk assessment [21], environmental management [22], and location selection [23]. ANP is a powerful method that has been applied to solve different decision-making problems by many researchers, and it is also applicable for constructing models with evaluation criteria and dimensions that contain complex interactions. Thus, ANP is selected as the appropriate tool to evaluate MRES performance in this study.

It should be considered that the input information of ANP is the subjective judgment information given by experts, which is often incomplete and hesitant due to the limitations of objective conditions and experts' knowledge structure. Therefore, it is particularly important to study how to express the expert judgment information and carry out decision-making scientifically. Fortunately, the probabilistic linguistic term sets (PLTS) provide a choice to express preferences by means of linguistic information. In practical applications, due to the qualitative natural of the judgment criteria, many linguistic and fuzzy methods have been developed. Rodriguez et al. [24] came up with hesitant fuzzy linguistic term sets (HFLTS) motivated by hesitant fuzzy sets [25] and linguistic term sets [26]. Based on this, Wei et al. [27] defined the operations on HFLTS. Chen et al. [28] investigated the consistency and consensus problems with the hesitant linguistic preference relations. Wang et al. [29] developed an optimization algorithm with the incomplete probabilistic linguistic term sets which could describe the qualitative pairwise judgment information in preference decision-making. These studies regarding probabilistic fuzzy or probabilistic linguistic provide fundamental theories for PLTS. As a method to model linguistic information, PLTS represents different membership degrees of all possible evaluation terms (linguistic terms) for a specific alternative, which is more likely to provide comprehensive and accurate preference information about the decision-makers.

Since the concept of PLTS was proposed while extending HFLTS, it has experienced substantial development, and it is now a hot topic in the field of multicriteria decision-making $[30,31]$. In recent years, many researchers have used the probabilistic linguistic information to solve complex decision-making problems. For example, Zhang et al. [32] introduced PLTS to describe group preferences while considering fuzzy and uncertain group preferences. Song et al. [33] proposed a novel text named Word2PLTS by introducing the idea of fuzziness and uncertainty of human language. Bai et al. [34] developed a new comparison method for PLTS to overcome the shortcoming of complex computing during PLTS application. Zhou et al. [35] used the ANP method under the probabilistic linguistic environment and integrated the PLTS and FTA-ANP. The combination of ANP and PLTS (PLTS/ANP for short) as the incomplete probabilistic linguistic ANP can make up for the shortcomings of the ANP method while using the PLTS method to represent the uncertain information. Based on this previous research, this study used PLTS/ANP to obtain the weights of MRES evaluation indexes and make an overall evaluation. In the process of obtaining the weights of MRES evaluation indexes by using the ANP method, the judgment about the important value of the evaluation index from experts might be fuzzy and hesitant due to the limitation of experts' knowledge or prejudice. At the time, the PLTS as a linguistic representation model with uncertainties can represent experts' hesitant information and give the values of 
a linguistic variable [30,36]. Moreover, PLTS can also reflect the associated probabilistic information about the linguistic terms, which achieves the objective of computing with expressions.

The motivation of this study is to develop an evaluation method for MRES by the integrated PLTS/ANP method and make a case study of 11 marine ranches cases in Rongcheng. MRES is a new concept, and it is very likely difficult for experts to have complete experience at this stage because of the lack of objective data and typical cases in this regard. Therefore, this study utilizes the integrated method of PLTS and ANP to evaluate MRES. This study focuses on the following three aspects:

(1) To construct an MRES evaluation network model based on the Driver-Pressure-State-Impact-Response (DPSIR) model, which includes not only evaluation indexes but also the influence relationships of indexes. The indexes for MRES involve economic, social, and ecological aspects, among which there are many complex interdependent relationships. The MRES evaluation network model shows the interdependencies that exist as inner dependence or outer dependence among clusters.

(2) To determine the prioritization of evaluation indexes by applying the PLTS/ANP method and calculate the evaluation value of MRES. This integrated PLTS/ANP method can reflect the influence mechanism between indexes and extract and integrate experts' judgment information reasonably, which is conducive for setting scientific priority weights. Combined with the weights calculated by the comprehensive method and the performance values of the marine ranching in each index, the comprehensive performances of MRES are obtained by weighted summation.

(3) To apply this proposed evaluation method to case study and conduct an effective assessment of marine ranching in Rongcheng. The evaluation value of MRES and the contribution rate of each cluster are calculated and analyzed, respectively, by using the index weight that is determined by PLTS/ANP and using the performance value of the case on each index. Finally, we give specific suggestions for improving ecosystem security for enterprises and government based on the results.

The remaining of this study is organized as follows. Section 2 constructs the evaluation index network model of the DPSIRbased MRES model and introduces the methodology of MRES evaluation using the integrated PLTS/ANP method. In Section 3, the MRES evaluation method, which integrates the PLTS and ANP, is applied to 11 marine ranches in the city of Rongcheng, and the evaluation results are analyzed as well. Based on the case study, some corresponding suggestions about MRES management improvement are given for these enterprises and government. Section 4 concludes the study with a discussion of the results in this study, the limitations of MRES evaluation by the integration method, and the future research direction.

\section{The PLTS/ANP-Based Evaluation Method for MRES}

This study utilizes the PLTS/ANP method to determine the evaluation framework for MRES and calculate the relative importance and prioritization of indexes. In other words, this study uses ANP to evaluate MRES while the PLTS provides a comprehensive way to represent complex linguistic information, so as to determine an accurate ranking of each evaluation index.

2.1. MRES Evaluation Framework with PLTS/ANP. The integrated PLTS/ANP used in this study to evaluate MRES includes the following steps. First, an evaluation index system is determined for MRES based on DPSIR. Second, the network model of MRES evaluation is constructed to show the influence relationships among evaluation indexes. Third, the overall weights of indexes are determined by PLTS/ANP. Fourth, the evaluation values of MRES are calculated and analyzed. The process is shown in Figure 1.

2.2. Evaluation Index System for MRES. The Organization for Economic Co-operation and Development (OECD) proposed the PSR model of environmental assessment in the late 1980s [37]. The United Nations (UN) adapted it for the DSR model [38], and then based on the advantages of the PSR and DSR models, the European Environment Agency (EEA) established the DPSIR framework and applied it to evaluate the relationship between environmental performance and social economy [39].

In this study, we construct the evaluation index system, which is an organic whole composed of multiple interrelated evaluation indexes from the five aspects of DPSIR for MRES. Specifically, in the evaluation system of MRES, the indexes of the driver cluster mainly come from the social system and the economic system, such as the profit margin and the enterprise's ecological awareness. The indexes of the pressure cluster mainly come from the ecological environment system and are directly affected by the driver, such as the green degree of farming methods and natural disasters. The indexes of the state cluster mainly represent the performance of the marine ranching ecological environment under pressure, such as water quality and target biomass. The indexes of the impact cluster mainly represent the effect of marine ranching on human social and economic life and the environment state, such as the improvement of fishery resources and the degree of pulling the industrial chain. The indexes of the response cluster mainly represent the positive measures taken by enterprises to improve the current situation in the process of operation and management, such as marine technology, and management and annual monitoring. 


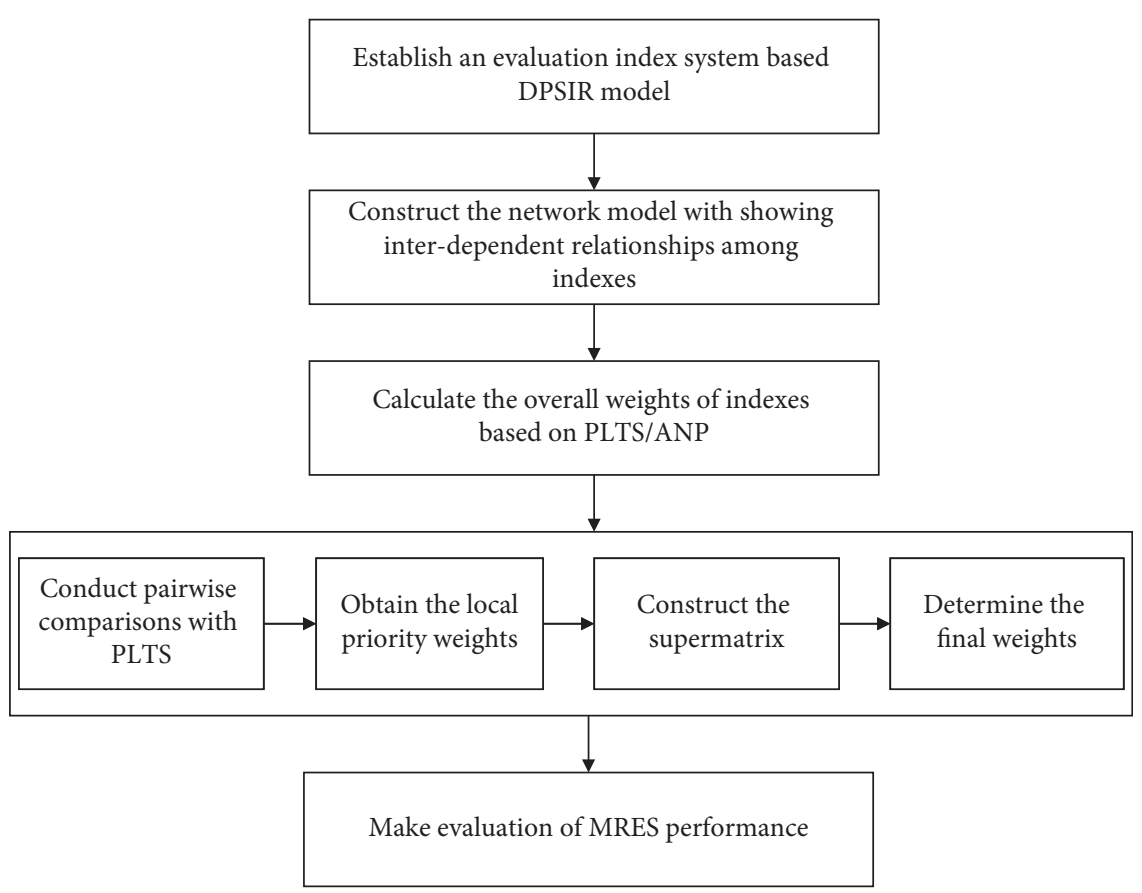

FIGURE 1: Framework of MRES evaluation.

The evaluation index system is established for MRES based on the DPSIR model, as shown in Table 1. From Table 1, it is obvious that the MRES index system comprises 5 clusters and 22 evaluation indexes. The evaluation indexes included in each cluster are carefully selected by referring to the relevant literature [40-42] and following the principles of being scientific, operative, forward-looking, and so on. In the attributes column of Table 1, the benefit-type index is defined as “+," and the cost-type index is defined as “-."

\subsection{Network Model Construction of MRES Evaluation.}

The ANP network structure is divided into control hierarchy and network hierarchy. Inside the network hierarchy, the network structure is composed of elements that are controlled by the control hierarchy, and they may interact with each other. In this study, the control hierarchy is omitted because it only contains one target element, namely MRES. During the process of ANP application, the network structure is constructed based on the identified indexes and clusters as well as their potential interrelationships. The interdependencies can exist in the form of inner dependence or outer dependence. Thus, an accurate modeling tool of comprehensive and interdependent indexes is provided.

As mentioned in Section 1, marine ranching is an artificial ecosystem, and the indexes listed in Table 1 may be interdependent and mutually influenced. There exist both outer dependency relationships and inner dependency relationships in the MRES evaluation system. (1) The outer dependency relationships may occur between the indexes from different clusters, and these relationships are also consistent with the correlations as shown by the DPSIR model [43]. For example, the evaluation index "enterprise's ecological awareness $\left(e_{3}\right)$ " from the "driver $\left(C_{1}\right)$ " cluster affects the evaluation index "green degree of farming methods $\left(e_{6}\right)$ " from the "pressure $\left(C_{2}\right)$ " cluster. Meanwhile, the evaluation index "research support $\left(e_{20}\right)$ " from the "response $\left(C_{5}\right)$ " cluster affects the evaluation index "profit margin $\left(e_{2}\right)$ " from the "driver $\left(C_{1}\right)$ " cluster. The same is true for the relationships between other different clusters. (2) The inner dependency relationships may occur between the indexes included in each cluster. For example, in the "impact $\left(C_{4}\right)$ " cluster, the index "the losses caused by natural or manmade disasters $\left(e_{16}\right)$ " affects another index "the improvement of fishery resources $\left(e_{13}\right)$," and the index "the improvement of fishery resources $\left(e_{13}\right)$ " has a positive effect on the index "the number of absorbed or resettled fishermen $\left(e_{14}\right)$." The same is true for the other four clusters.

Based on the above analysis, according to the DPSIR model, this study constructs the network model of MRES evaluation, as shown in Figure 2. In this network model, arrows are used to represent the influence relationships inside or outside the cluster, and arrows point one object to other objects that are affected.

2.4. Determination of Overall Weights of Indexes. In the existing literature, the evaluation indexes of marine ranching are based on assuming that the indexes are independent of each other. In fact, marine ranching is an ecosystem, and its MRES evaluation system exhibits the influence relationships, as shown in Figure 2. A key problem is how to reflect such influence relationships and make a reasonable evaluation. Due to the complexity of MRES, the research and understanding of some evaluation indexes are limited at present. It is difficult for experts to express preferences by means of one certain linguistic term. Therefore, this study first uses the PLTS method to effectively achieve the uncertain 
TABLE 1: MRES evaluation index system.

\begin{tabular}{|c|c|c|c|}
\hline Cluster & Evaluation index & Unit & Attributes \\
\hline \multirow{3}{*}{ Driver $\left(C_{1}\right)$} & Financial fund input $\left(e_{1}\right)$ & $10^{4}$ yuan & + \\
\hline & Profit margin $\left(e_{2}\right)$ & $\%$ & + \\
\hline & Enterprise's ecological awareness $\left(e_{3}\right)$ & $\%$ & + \\
\hline \multirow{5}{*}{ Pressure $\left(C_{2}\right)$} & The number of bottom sowing and proliferation and release $\left(e_{4}\right)$ & $10^{4}$ tails & + \\
\hline & Seaweed field and seagrass bed transplant cultivation $\left(e_{5}\right)$ & Number & + \\
\hline & Green degree of farming methods $\left(e_{6}\right)$ & Number & + \\
\hline & Artificial reef construction and maintenance $\left(e_{7}\right)$ & Number & + \\
\hline & Natural disaster $\left(e_{8}\right)$ & Times/year & - \\
\hline \multirow{4}{*}{ State $\left(C_{3}\right)$} & Water quality $\left(e_{9}\right)$ & Number & - \\
\hline & Marine sediment $\left(e_{10}\right)$ & Number & - \\
\hline & Target biomass $\left(e_{11}\right)$ & Number & + \\
\hline & Biodiversity index $\left(e_{12}\right)$ & Number & + \\
\hline \multirow{5}{*}{ Impact $\left(C_{4}\right)$} & The improvement of fishery resources $\left(e_{13}\right)$ & Number & + \\
\hline & The number of absorbed or resettled fishermen $\left(e_{14}\right)$ & Number & + \\
\hline & The degree of pulling the industrial chain $\left(e_{15}\right)$ & $10^{4}$ yuan & + \\
\hline & The losses caused by natural or man-made disasters $\left(e_{16}\right)$ & $10^{4}$ yuan & - \\
\hline & Benefits of aquatic products $\left(e_{17}\right)$ & $\%$ & + \\
\hline \multirow{5}{*}{ Response $\left(C_{5}\right)$} & Visualization, intelligence, information system construction $\left(e_{18}\right)$ & Number & + \\
\hline & Marine technology and management $\left(e_{19}\right)$ & Number & + \\
\hline & Research support $\left(e_{20}\right)$ & Number & + \\
\hline & Scientific management of fishery resources $\left(e_{21}\right)$ & Number & + \\
\hline & Annual monitoring $\left(e_{22}\right)$ & Times/year & + \\
\hline
\end{tabular}

preferences given by experts and then uses ANP to make an overall evaluation for the MRES. The associated calculation for MRES consists of the following steps.

Step 1: conduct the pairwise comparisons with PLTS. The definition of ANP is that as a multicriteria theory, it provides the mathematics and a comprehensive structure to obtain the relative influence of one of two elements over the other in a pairwise comparison process on a third element in the system, with respect to an underlying control criterion [16]. As the ANP method has been described sufficiently in the literature, it is described very briefly in this section. For the complete process or model of constructing pairwise comparisons, see this cited article of Saaty [15].

Based on Figure 2, ANP conducts pairwise comparisons that reflect dependencies in this network model of MRES evaluation between all clusters and indexes.

For each relationship between clusters $C_{i} \longrightarrow C_{j}$ in the network structure as shown in Figure 1, the pairwise comparison matrices should be constructed. Obviously, if $i \neq j, C_{i} \longrightarrow C_{j}$ represents the outer dependent relationships between two different clusters; if $i=j$, it represents the inner dependent relationships in one cluster. Without loss generality, here, we suppose $C_{i}=\left\{e_{i 1}, e_{i 2}, \ldots, e_{i M_{i}}\right\}$ and $C_{j}=\left\{e_{j 1}, e_{j 2}, \ldots, e_{j N_{j}}\right\}$. Following the thought of ANP, the element/index $e_{i m}$ included in cluster $C_{i}$ is regarded as a criterion, and then, a pairwise comparison matrix $B_{j}^{i m}$ of cluster $C_{j}$ is constructed with respect to $e_{i m}$.

Here, we give some concepts and definitions of PLTS and show the process for constructing $B_{j}^{i m}$ with PLTS [44].

The most widely used concept about PLTS is the linguistic term set (LTS), which can be defined as in

$$
S=\left\{s_{0}, s_{1}, \ldots, s_{\tau}, \ldots, s_{2 \tau}\right\}
$$

where $2 \tau+1$ is the number of all terms in $S$; $s_{\delta}(\delta \in[0,2 \tau])$ as the linguistic term is generated by a predefined syntactic rule and restricted by a fuzzy set; $s_{\tau}$ means "indifference", and the remaining linguistic terms are distributed symmetrically around it.

Then, the PLTS is defined as in

$$
L(p)=\left\{L^{(\ell)}\left(p^{(\ell)}\right) \mid L^{(\ell)} \in S, \quad p^{(\ell)} \geq 0, k=1,2, \ldots, \# L(p), \sum_{\ell=1}^{\# L(p)} p^{(\ell)} \leq 1\right\}
$$

where $L^{(\ell)}\left(p^{(\ell)}\right)$ is the $\ell$ th linguistic term of $L^{(\ell)}$ with the associated probability $p^{(\ell)} ; \# L(p)$ is the number of all different elements in $L(p)$. Similarly, we utilize $S$ to evaluate these indexes and to present the preference degree for an index $e_{j}$ over $e_{j^{\prime}}$ in a pairwise comparison matrix $B_{j}^{i m}$. 


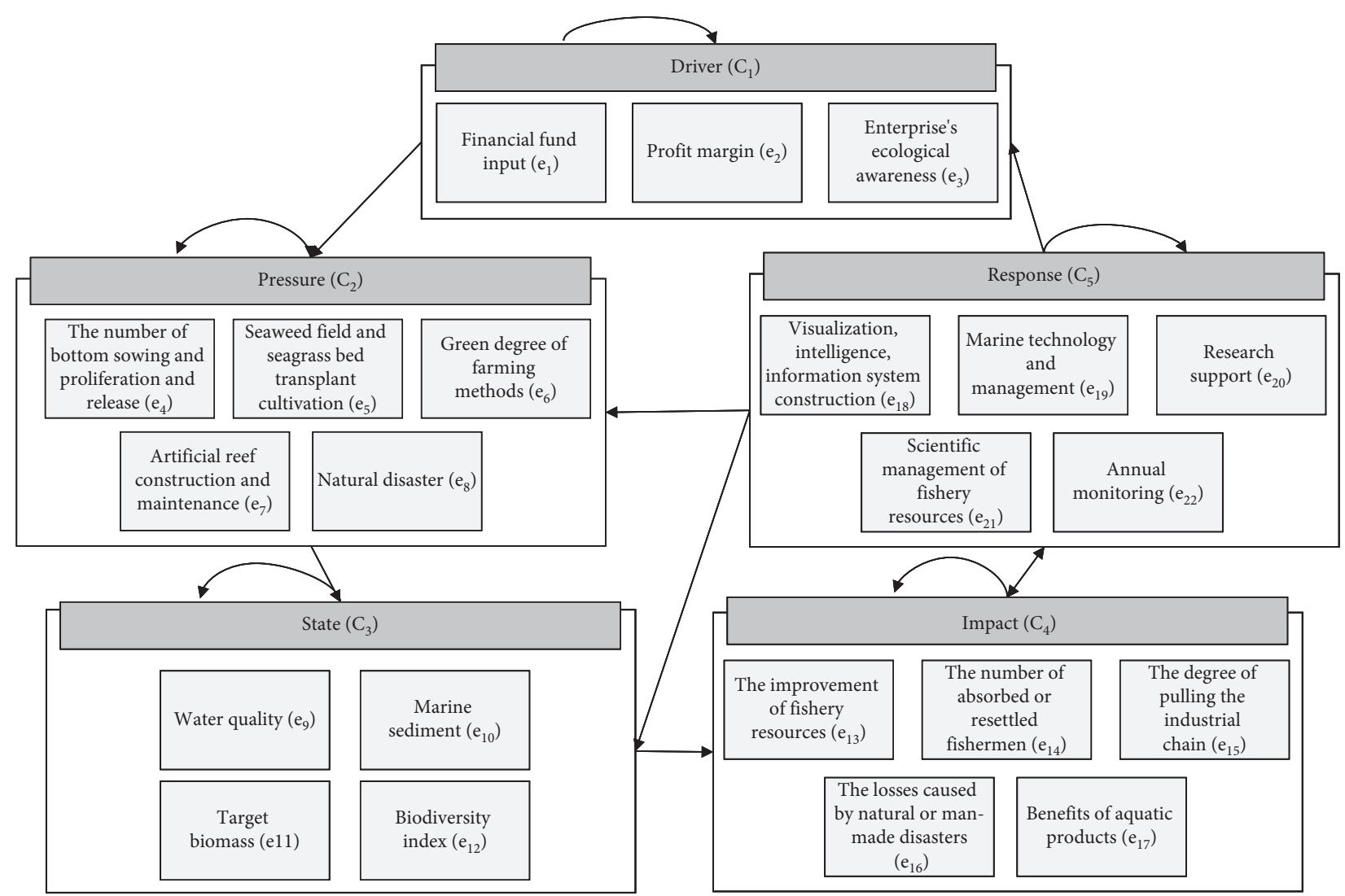

FIGURE 2: Network model of MRES evaluation.

$$
\begin{aligned}
B_{j}^{i m} & =\left(L_{k k^{\prime}}(p)\right)_{N_{j} \times N_{j}}, \\
L_{k k^{\prime}}(p) & =\left\{L_{k k^{\prime}}^{(\ell)}\left(p_{k k^{\prime}}^{(\ell)}\right) \mid \ell=1,2, \ldots, \# L_{k k^{\prime}}(p)\right\},
\end{aligned}
$$

where $p_{k k^{\prime}}^{(\ell)}>0$ and $\# L_{k k^{\prime}}(p)$ are the number of linguistic terms in $L_{k k^{\prime}}(p)$. Moreover, there are some rules that need to be followed while constructing a matrix $B_{j}^{i m}$, such as $p_{k k^{\prime}}^{(\ell)}=p_{k^{\prime} k}^{(\ell)}, L_{k k^{\prime}}^{(\ell)}+L_{k^{\prime} k}^{(\ell)}=s_{2 \tau}, L_{k k}(p)=$ $s_{\tau}(1)$, and $\# L_{k k^{\prime}}(p)=\# L_{k^{\prime} k}(p)$.

Step 2: obtain the local priority weights.

For a PLTS, the weighted value of the $\ell$ th element in the PLTS is defined as

$$
W V^{\ell}=I\left(L^{(\ell)}\right) \times p^{(\ell)},
$$

where $\operatorname{Ind}(\cdot)$ is a function that returns the subscript of a linguistic term from $S$ to $[0,2 \tau]$, for example, $\operatorname{Ind}\left(s_{\delta}\right)=$ $\delta$ for any $s_{\delta} \in S$.

For the pairwise matrix $B_{j}^{i m}$ expressed by probabilistic linguistic terms, if $B_{j}^{i m}$ is consistent, motivated by reference [45], we can get the most accurate weights of elements by the optimization model as in

$$
\left\{\begin{array}{l}
\min Z_{j}^{i m}=\sum_{k=1}^{N_{j}} \sum_{k^{\prime}=1}^{N_{j}} \sum_{\ell=1}^{\# L_{i j}}\left(\varepsilon_{k k^{\prime}}^{\ell}\right)^{2}=\sum_{k=1}^{N_{j}} \sum_{k^{\prime}=1}^{N_{j}} \sum_{\ell=1}^{\# L_{i j}}\left(\ln \omega_{k}-\ln \omega_{k^{\prime}}-\ln \frac{W V_{k k^{\prime}}^{\ell}}{W V_{k^{\prime} k}^{\ell}}\right)^{2} \\
\text { s.t. } \sum_{k=1}^{N_{j}} \omega_{k}=1, \quad \omega_{k}>0, k=1,2, \ldots, N_{j}
\end{array}\right.
$$

where $\varepsilon_{k k^{\prime}}^{\ell}=\ln \omega_{k}-\ln \omega_{k^{\prime}}-\ln \left(W V_{k k^{\prime}}^{\ell} / W V_{k^{\prime} k}^{\ell}\right), \quad \ell \in$ $\left.1,2, \ldots, \# L_{i j}\right\} ; \omega_{k}$ is the weight of the kth index corresponding to matrix $B_{j}^{i m}, k=1,2, \ldots, N_{j}$. 
As proved in reference [4], the optimal solution of the model as in equation (5) can be calculated by

$$
\omega_{k}=\left\{\begin{array}{l}
\frac{\exp \left(q_{k}\right)}{\sum_{k^{\prime}=1}^{N_{j}-1} \exp \left(q_{k^{\prime}}\right)+1}, k=1,2, \ldots, N_{j}-1, \\
\frac{1}{\sum_{k=1}^{N_{j}-1} \exp \left(q_{k}\right)+1}, k=N_{j} .
\end{array}\right.
$$

In equation (6), $\exp (\cdot)$ is the exponential function based on the natural constant $e \approx 2.71828$, with $Q=\left(q_{1}, q_{2}, \ldots, q_{N_{j}-1}\right)=D^{-1} Y$, in which

$$
\begin{aligned}
& D=\left(\begin{array}{cccc}
\sum_{k=2}^{N_{j}} \ell_{1 k} & -\ell_{12} & \cdots & -\ell_{1\left(N_{j}-1\right)} \\
-\ell_{21} & \sum_{\substack{k=1 \\
k \neq 2}}^{N_{j}} \ell_{2 k} & \cdots & -\ell_{2\left(N_{j}-1\right)} \\
\vdots & \vdots & \ddots & \vdots \\
-\ell_{\left(N_{j}-1\right) 1} & -\ell_{\left(N_{j}-1\right) 2} & \cdots & \sum_{\substack{k=1 \\
k \neq N_{j}-1}}^{N_{j}} \ell_{\left(N_{j}-1\right) k}
\end{array}\right), \\
& Y=\left(\begin{array}{c}
\sum_{k=1}^{N_{j}} \sum_{\ell=1}^{\# L_{1 j}} \ln \frac{W V_{1 k}^{\ell}}{W V_{k 1}^{\ell}} \\
\sum_{k=1}^{N_{j}} \sum_{\ell=1}^{\# L_{2 j}} \ln \frac{W V_{2 k}^{\ell}}{W V_{k 2}^{\ell}} \\
\vdots \\
\sum_{k=1}^{N_{j}} \sum_{\ell=1}^{\# L}\left(N_{j-1}^{\left(N_{j}\right) k} \frac{W V_{\left(N_{j}-1\right) k}^{\ell}}{W V_{k\left(N_{j}-1\right)}^{\ell}}\right.
\end{array}\right) .
\end{aligned}
$$

Step 3: construct the supermatrix.

Using Steps 1 and 2, the vector of local priority weights $\left(\omega_{j}^{i m}=\left(\omega_{1}, \omega_{2}, \ldots, \omega_{N_{j}}\right)\right)$ corresponding to the matrix $B_{j}^{i m}$ is determined. To reflect the relationship between local priority weights and its corresponding criterion, here, the vector of local priority weights is redescribed as $\omega_{j}^{i m}=\left(\omega_{1}^{i m}, \omega_{2}^{i m}, \ldots, \omega_{N_{j}}^{i m}\right)$ by adding the superscript into the symbols. Similarly, also for each relationship between clusters $C_{i} \longrightarrow C_{j}$, taking another element (index) $e_{i m^{\prime}}$ included in cluster $C_{i}$ as a criterion, the pairwise comparison matrix $B_{j}^{i m^{\prime}}$ of cluster $C_{j}$ is constructed with respect to $e_{i m^{\prime}}$, and its corresponding vector of local priority weights $\omega_{j}^{i m^{\prime}}=\left(\omega_{1}^{i m^{\prime}}, \omega_{2}^{i m^{\prime}}, \ldots, \omega_{N_{j}}^{i m^{\prime}}\right)$ can also be determined, $m^{\prime} \neq m \in\left\{1,2, \ldots, M_{i}\right\}$. Consequently, all of the local priority weights for $C_{i} \longrightarrow C_{j}$ can be written as a block matrix $W_{i j}$, as in equation (9). The block matrix $W_{i j}$ represents the relative importance of the elements in cluster $C_{j}=\left\{e_{j 1}, e_{j 2}, \ldots, e_{j N_{j}}\right\}$ with regard to each element in cluster $C_{i}=\left\{e_{i 1}, e_{i 2}, \ldots, e_{i M_{i}}\right\}$ [46].

$$
W_{i j}=\left[\begin{array}{cccc}
\omega_{1}^{i 1} & \omega_{1}^{i 2} & \cdots & \omega_{1}^{i M_{i}} \\
\omega_{2}^{i 1} & \omega_{2}^{i 2} & \cdots & \omega_{2}^{i M_{i}} \\
\vdots & \vdots & \ddots & \vdots \\
\omega_{N_{j}}^{i 1} & \omega_{N_{j}}^{i 2} & \cdots & \omega_{N_{j}}^{i M_{i}}
\end{array}\right] .
$$

For each pair of clusters in Figure 1, if there exists a relationship between clusters $C_{i} \longrightarrow C_{j}$, then the block matrix $W_{i j}$ is determined by equations (4)-(9); else if there does not exist a relationship between clusters $C_{i} \longrightarrow C_{j}$, then the block matrix $W_{i j}$ is defined by zero matrix $\left(W_{i j}=0\right)$. When all of the block matrices are 
determined, a supermatrix in the ANP is constructed as in equation (10) to reflect all the local priority weights in the network model of MRES evaluation [47]. Note that, the number of clusters in the MRES evaluation is 5 resulting in there are $5 \times 5=25$ block matrices in the supermatrix $W$.

$$
\begin{aligned}
W & =\left[W_{i j}\right]_{5 \times 5} \\
& =\left[\begin{array}{cccc}
W_{11} & W_{12} & \cdots & W_{15} \\
W_{21} & W_{22} & \cdots & W_{25} \\
\vdots & \vdots & \ddots & \vdots \\
W_{51} & W_{52} & \cdots & W_{55}
\end{array}\right] .
\end{aligned}
$$

Step 4: determine the final weights.

The supermatrix $W$, as in equation (10), is unweighted since the sum of elements in each column is not equal to 1 . In order to normalize the supermatrix, the weight of each block matrix is determined by experts according to the ANP [8]. Assuming the determined weight of block matrix $W_{i j}$ is $a_{i j}, \sum_{i=1}^{5} a_{i j}=1, a_{i j} \geq 0$, $j=1,2, \ldots, 5$, then the weighted supermatrix $\bar{W}$ is calculated by integrating the weight of block matrix into the supermatrix as in

$$
\begin{aligned}
\bar{W} & =\left[\bar{W}_{i j}\right]_{5 \times 5} \\
& =\left[\begin{array}{cccc}
a_{11} W_{11} & a_{12} W_{12} & \cdots & a_{15} W_{15} \\
a_{21} W_{21} & a_{22} W_{22} & \cdots & a_{25} W_{25} \\
\vdots & \vdots & \ddots & \vdots \\
a_{51} W_{51} & a_{52} W_{52} & \cdots & a_{55} W_{55}
\end{array}\right] .
\end{aligned}
$$

The limit weighted supermatrix that reflects both direct influence relationships and indirect influence relationships between indexes is calculated by $\bar{W}^{\infty}=\lim _{\lambda \rightarrow \infty} \bar{W}^{2 \lambda+1}$, as in equation (12).

According to the ANP theory, the weighted supermatrix $\bar{W}$ is a column random matrix, that is, the sum of each column is equal to 1 . Thus, the limit weighted supermatrix $\bar{W}^{\infty}$ must be stabilized, that is, the elements in each row are equal to each other such as $w_{n 1}=w_{n 2}=\cdots=w_{n N}$, $n=1,2, \ldots, N$. For convenience, here we suppose $w_{n}=w_{n 1}=w_{n 2}=\cdots=w_{n N}$, and it is the global priority weight of index $e_{n}, n=1,2, \ldots, N$. Obviously, the number of indexes in the MRES evaluation is 22 as shown in Table 1 resulting in $N=22$ in the limit weighted supermatrix $\bar{W}^{\infty}$.

$$
\begin{aligned}
\bar{W}^{\infty} & =\left[w_{n n^{\prime}}\right]_{N \times N} \\
& =\left[\begin{array}{cccc}
w_{11} & w_{12} & \cdots & w_{1 N} \\
w_{21} & w_{22} & \cdots & w_{2 N} \\
\vdots & \vdots & \ddots & \vdots \\
w_{N 1} & w_{N 2} & \cdots & w_{N N}
\end{array}\right] .
\end{aligned}
$$

2.5. Calculation of the Evaluation Value of MRES. We suppose that there are $T$ marine ranches to be evaluated on the MRES, and the performance value of the $t$ th marine ranching $M R_{t}$ on the nth index $e_{n}$ be $x_{t n} ; t=1,2, \ldots, T$, $n=1,2, \ldots, 22$. As mentioned in Table 1 , the evaluation indexes are divided as the benefit-type index marked with “+," and the cost-type index marked with “-." In order to eliminate dimensional and index-type effects, the collected index data of marine ranching $X=\left[x_{t n}\right]_{T \times 22}$ need to be standardized before calculation by equations (13) and (14). Equation (13) is used to standardize the data on benefit index "+," and equation (14) is used to standardize that on the cost index “-”.

$$
\begin{aligned}
y_{t n} & =\frac{x_{t n}-\min \left\{x_{1 n}, \ldots, x_{T n}\right\}}{\max \left\{x_{1 n}, \ldots, x_{T n}\right\}-\min \left\{x_{1 n}, \ldots, x_{T n}\right\}}, \\
y_{t n} & =\frac{\max \left\{x_{1 n}, \ldots, x_{T n}\right\}-x_{t n}}{\max \left\{x_{1 n}, \ldots, x_{T n}\right\}-\min \left\{x_{1 n}, \ldots, x_{T n}\right\}} .
\end{aligned}
$$

After the collected data are all standardized, the comprehensive MRES evaluation value (MRES value for short) can be obtained by combining the index weights with the standardized performance data of the marine ranching, as in equation (15). The higher the value of $Q_{t}$ in the calculation result, the better the MRES of the marine ranching $M R_{t}$.

$$
Q_{t}=\sum_{n=1}^{22} y_{t n} w_{n}, t=1,2, \ldots, T .
$$

For better analysis, the MRES is usually divided into several grade levels according to comprehensive MRES evaluation values. Similar to references $[8,48]$, the grades of the MRES in this study are divided into three levels of insecurity, medium security, and security. The MRES is obtained according to the following steps:

Step 1: finding the maximum value $Q_{\max }$ and the minimum value $Q_{\min }$ of MRES from $Q_{t}, t=1,2, \ldots, T$.

Step 2: calculating the distance between the maximum and the minimum values $\Delta=Q_{\max }-Q_{\min }$.

Step 3: determining the grade level of the marine ranching $M R_{t}$ by equation (16), $t=1,2, \ldots, T$.

$$
Q_{t}=\left\{\begin{array}{l}
{\left[Q_{\min }, Q_{\min }+\frac{\Delta}{3}\right), \text { in security, }} \\
{\left[Q_{\min }+\frac{\Delta}{3} Q_{\max }-\frac{\Delta}{3}\right), \text { medium - security, }} \\
{\left[Q_{\max }-\frac{\Delta}{3}, Q_{\max }\right], \text { security. }}
\end{array}\right.
$$

The comprehensive MRES evaluation value is composed of the performance in five clusters, that is, $Q_{t}=\sum_{i=1}^{5} Q_{t}^{i}$, 
where $Q_{t}^{i}=\sum_{n=1}^{M_{i}} w_{n} \cdot y_{t i}, t=1,2, \ldots, T, M_{i}$ is the number of indexes included in the cluster $C_{i}$. Consequently, the contribution rate is defined, as in equation (17), to reflect the contribution of a cluster to the MRES.

$$
C R_{t}^{i}=Q_{t}^{i} / Q_{t}, i=1,2, \ldots, 5, t=1,2, \ldots, T .
$$

The higher the value of $C R_{t}^{i}$, the greater the contribution rate of cluster $C_{i}$ to the MRES for $M R_{t}$. It means that the marine ranching $M R_{t}$ has the advantage on the cluster with a greater contribution rate. If marine ranching $M R_{t}$ wants to improve its MRES level, it can start from the cluster (indexes) with a lower contribution rate. Furthermore, the average contribution rate is easily calculated by $C R_{i}=\sum_{t=1}^{T} C R_{t}^{i} / T$ to reflect the average level of several marine ranches, $i=1,2, \ldots, 5$.

\section{Case Study for Marine Ranching in Rongcheng}

3.1. Research Area and Date Source. Located in Weihai, Shandong Province, Rongcheng has $500 \mathrm{~km}$ of coastline. Its marine ranches occupy an area of $313 \mathrm{~km}^{2}$. As of January 2021, Shandong had 54 national marine ranches, accounting for $39.7 \%$ of the national marine ranching programs in China. Among them, Rongcheng has successfully established 10 national marine ranches and 14 provincial marine ranches, making fruitful achievements in the construction of marine ranching. In the sixth batch of the national marine ranching demonstration list published by the Ministry of Agriculture and Rural Affairs of the People's Republic of China, Rongcheng ranked first among county-level cities in China regarding the number of newly added ranches. To assess the MRES, this study collects data from 11 typical marine ranches in Rongcheng, which consist of eight national marine ranches $\left(\mathrm{MR}_{1}-\mathrm{MR}_{8}\right)$ and three provincial marine ranches $\left(\mathrm{MR}_{9}-\mathrm{MR}_{11}\right)$. We use the index system and evaluation method constructed above for analysis. The location of the study area is shown in Figure 3.

\subsection{Results and Analysis}

3.2.1. Index Priority Weights. As mentioned in Sections 2.1-2.4, the index weights of MRES evaluation are determined by the integrated PLTS/ANP method. First, the pairwise matrices are constructed, which reflect the connection relationships between elements and clusters according to Figure 2. Then, experts give all the pairwise comparison matrices, in which the probabilistic linguistic terms are used as needed to represent uncertain information. In this study, the set of linguistic term $S$ is defined with $\tau=8$. The key linguistic terms are $s_{0}=$ extremely more unimportant, $s_{2}=$ very strongly unimportant, $s_{4}=$ strongly more unimportant, $s_{6}=$ moderately more unimportant, $s_{8}=$ equally important, $s_{10}=$ moderately more important, $s_{12}=$ strongly more important, $s_{14}=$ very strongly important, and $s_{16}=$ extremely more important. The linguistic terms $s_{1}, s_{3}$, $s_{5}, s_{7}, s_{9}, s_{11}, s_{13}$, and $s_{15}$ are the intermediate terms between the $s_{i-1}$ and $s_{i+1}$. Finally, we put the experts' information into equations (4)-(8) to obtain the vector of local priority weights of MRES evaluation indexes.

An example of the pairwise comparison matrices is shown in Table 2, which illustrates the vector of local priority weights corresponding to cluster $C_{5}$ with respect to the index $e_{6}$ in cluster $C_{2}$. In Table 2 , the data " $s_{4}(0.8), s_{3}(0.2)$," which are in the second row and the third column, show that the relatively important degree of $e_{19}$ to $e_{21}$ with respect to $e_{6}$ is strongly more unimportant $\left(s_{4}\right)$ with 0.8 probability and $\left(s_{3}\right)$ with 0.2 probability. The final global priority weights of indexes (index weights for short) can be determined by calculating the supermatrix, weighted supermatrix, and limit-weighted supermatrix as shown in equations (9)-(12). The results are shown in Figure 4.

At the index level, the weight of an index reflects its importance degree relative to all the indexes from the evaluation index system. For an evaluation index, the higher the weight, the more impact it has on the MRES. According to Figure 4, "scientific management of fishery resources $\left(e_{21}\right)$ " is the first priority index among all the evaluation indexes, with a weight of 0.1142 . This means that the scientific management of fishery resources is the most critical index for evaluating MRES. Other indexes that have high weights (above 0.06 ) are profit margin (0.1017), enterprise's ecological awareness (0.0823), the degree of pulling the industrial chain $(0.0781)$, the improvement of fishery resources (0.0639), and benefits of aquatic products (0.0609). This indicates that these indexes are of great importance to MRES. The number of absorbed or resettled fishermen (0.0050) and the number of bottom sowing and proliferation and release (0.0079) are relatively less influential indexes in order of importance with weights less than 0.01 .

At the clusters level, according to Figure 4, the weights of clusters are ranked in the following order: response (0.2646) $>$ impact $\quad(0.2350)>$ driver $\quad(0.21465)>$ state $\quad(0.1772)$ $>$ pressure (0.1047). This shows that the five clusters from the evaluation model based on DPSIR have different influences on MRES, with the response cluster having the largest effect and the pressure cluster having the lowest effect. Response, impact, and state are the clusters with weights between 0.1772 and 0.2350 .

For each cluster, the weights of its included indexes reflect the different influences on MRES within the same cluster. In the driver cluster, profit margin (0.1017) has the highest weight, followed by financial fund input (0.0345) and enterprise's ecological awareness (0.0823). In the pressure cluster, the weight of natural disaster (0.0494) is significantly higher than the remaining four indexes, whose weights are not greater than 0.021 , reflecting the spacing of indexes in the one cluster. In the state cluster, the weight values of biodiversity index (0.0548), water quality (0.0530), and target biomass $(0.0514)$ are very close, and marine sediment with the weight of 0.0180 is relatively small. In the impact cluster, the degree of pulling the industrial chain (0.0781) has the highest weight, which has an obvious difference to the number of absorbed or resettled fishermen (0.0050), which is the lowest weight in this cluster. In the response cluster, scientific management of fishery resources $(0.1142)$ has the highest weight, and annual monitoring $(0.0147)$ has the lowest. Visualization, intelligence, 


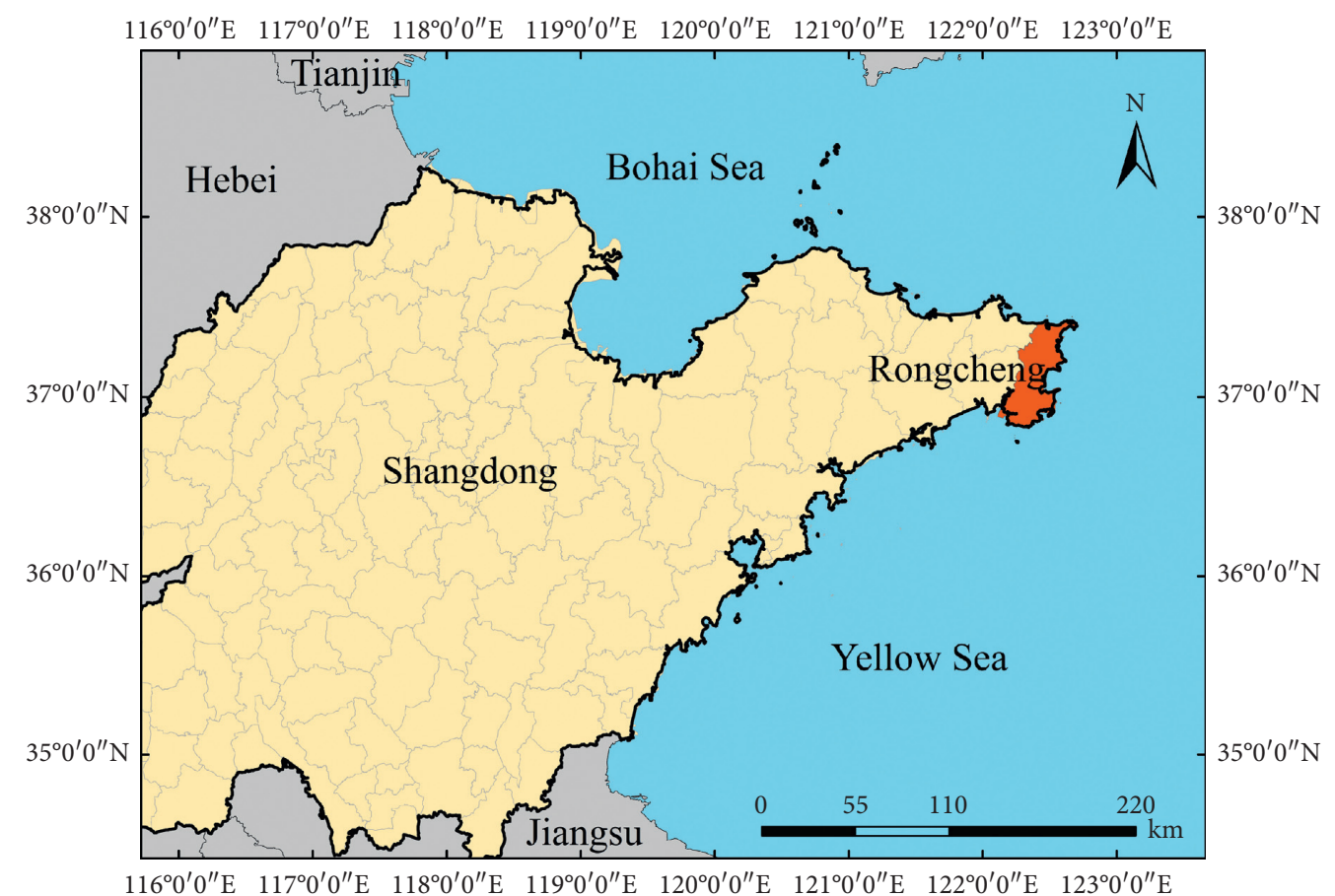

Figure 3: Geographical location of the research area of this study.

Table 2: A pairwise comparison matrix for cluster $C 5$ with respect to index $e 6$.

\begin{tabular}{lcccc}
\hline$e_{6}$ & $e_{19}$ & $e_{21}$ & $e_{22}$ & Priority vector \\
\hline$e_{19}$ & $s_{8}(1)$ & $s_{4}(0.8), s_{3}(0.2)$ & $s_{10}(0.7), s_{11}(0.3)$ & 0.1795 \\
$e_{21}$ & $s_{12}(0.8), s_{13}(0.2)$ & $s_{8}(1)$ & $s_{14}(0.9), s_{15}(0.1)$ & 0.7383 \\
$e_{22}$ & $s_{6}(0.7), s_{5}(0.3)$ & $s_{2}(0.9), s_{1}(0.1)$ & $s_{8}(1)$ & 0.0822 \\
\hline
\end{tabular}

information system construction (0.0314), marine technology and management (0.0483), and research support (0.0560) have different levels of influence.

\subsubsection{Overall Evaluation Value of MRES. As mentioned in} Section 2.5, based on the index weights, the comprehensive evaluation value of MRES, which is used to reflect the final performance results of the marine ranches on ecological security, can be calculated by equations (13)-(15). Inputting the index weights calculated in Section 3.2.1 and the standardized data of the investigated 11 marine ranches in Rongcheng into equation (15), we can calculate the comprehensive evaluation values of MRES, as shown in Figure 5. The average performances of the national and provincial marine ranches with respect to MRES are obtained by calculating the mean of the MRES values of national and provincial marine ranches, respectively.

In addition, the grades of the MRES should be divided into three levels by following the three steps in Section 2.5, and each level can be determined by equation (16). If the MRES evaluation value falls within the range of $[0.48,0.58]$, it is defined as grade 1 , namely insecurity. If the MRES evaluation value falls within the range of [0.58, 0.67], it is defined as grade 2 , namely medium security. If the MRES evaluation value falls within the range of $[0.67,0.76]$, it is defined as grade 3 , namely security. The grade levels of the MRES shown in Figure 5 reflect the security or sustainability degree of each of the 11 marine ranches operating in Rongcheng.

It is obvious that all MRES evaluation values of the 11 marine ranches range between 0.48 and 0.76. Among the evaluation values of the 11 marine ranches, four are in the insecurity grade, namely $\mathrm{MR}_{1}, \mathrm{MR}_{2}, \mathrm{ME}_{3}$, and $\mathrm{MR}_{9}$; three of them are in the medium security grade, namely $\mathrm{MR}_{11}, \mathrm{MR}_{4}$, and $\mathrm{MR}_{6}$; four of them are in the security grade, namely $\mathrm{MR}_{8}$, $\mathrm{MR}_{10}, \mathrm{MR}_{7}$, and $\mathrm{MR}_{5}$. These evaluation results indicate that there are differences in the MRES performance of marine ranches in Rongcheng, and the development process of marine ranching is inconsistent in terms of ecological security. In other words, we can see that $36 \%$ of the marine ranches have relatively good performance in ecological security; $28 \%$ of them have average performance and have room to improve ecological security degree; and $36 \%$ of them have poor performance, so they need to be improved promptly. In particular, to find the underlying problems of the marine ranches, we conducted in-depth analysis from the national and provincial perspectives.

According to Figure 5, among the eight national marine ranches $\left(\mathrm{MR}_{1}-\mathrm{MR}_{8}\right), 37.5 \%$ of the marine ranches are in the security grade; $25 \%$ are in the medium security grade; and $37.5 \%$ are in the insecurity grade. Overall, the average MRES value of the national marine ranches is 0.61 , which is in the 


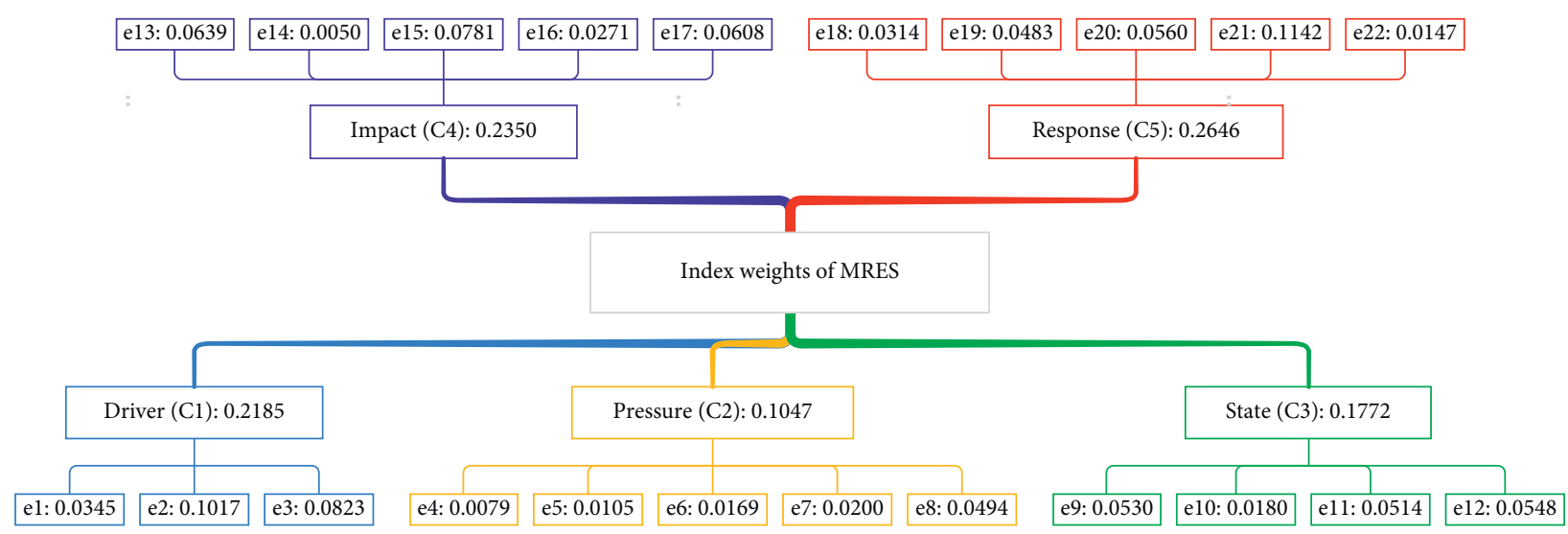

Figure 4: Index weights of MRES.

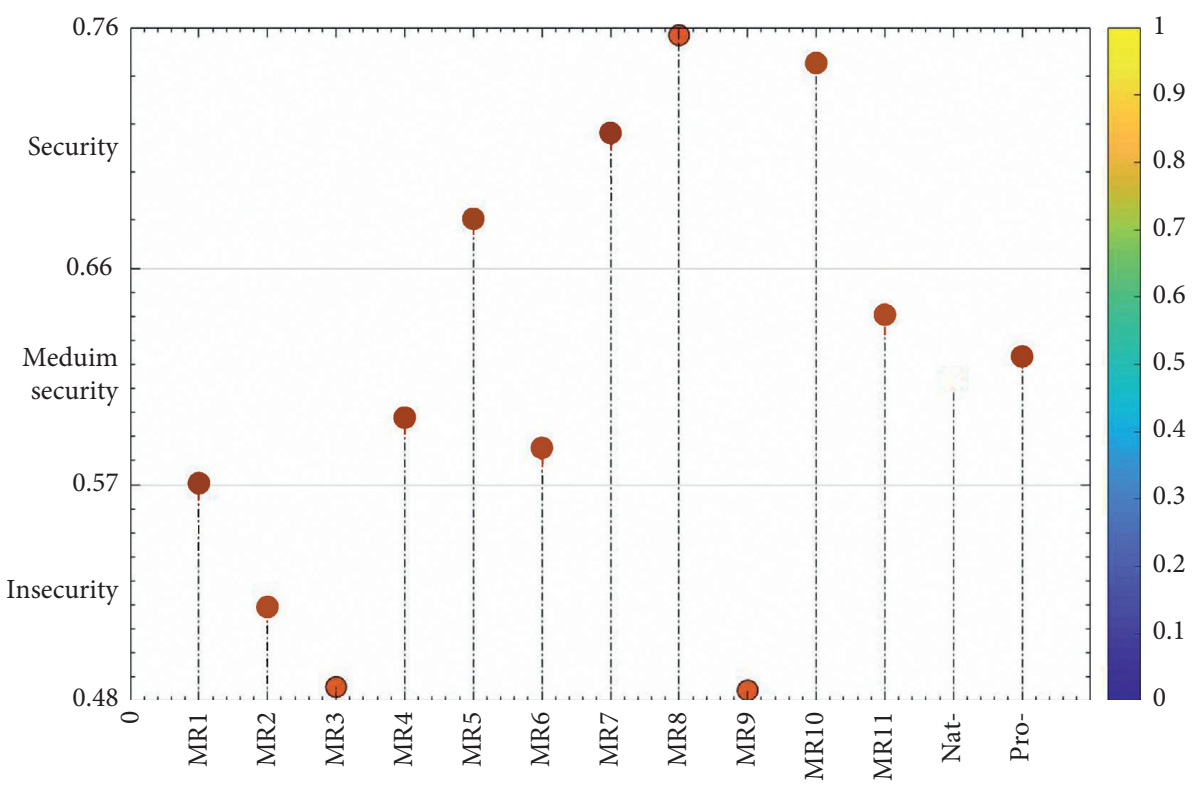

FIGURE 5: MRES evaluation values for marine ranches in Rongcheng.

medium security grade. It indicates that although a lot of efforts for pursuing the sustainable development of marine ranching and preserving the marine ecological environment have been made, for national marine ranching, there is still a gap between the current ecological security status and the ideal state.

Among the three provincial marine ranches $\left(\mathrm{MR}_{9}-\mathrm{MR}_{11}\right), 33.3 \%$ are in the security grade; $33.3 \%$ are in the medium security grade; and $33.3 \%$ are in the insecurity grade. Overall, the average MRES value of the provincial marine ranches is 0.62 , which is in the medium security grade. Therefore, the evaluation grade level of MRES indicates that the ecological security of provincial marine ranching also has a large room for improvement as only $33.3 \%$ of the marine ranches are in the security grade. The maximum and minimum values of MRES in provincial marine ranches are 0.75 of $\mathrm{MR}_{10}$ and 0.48 of $\mathrm{MR}_{9}$, and they are also the maximum and minimum values of MRES in the 11 marine ranches in this study, which indicates that the ecological security grade of provincial marine ranching is seriously uneven.
By comparing national and provincial marine ranching, we note that the MRES performance of national and provincial marine ranching does not show an obvious correlation with the rating level of marine ranching (i.e., national level or provincial level). First, for either national or provincial marine ranches, the distribution of marine ranching on the MRES grade level is similar, with no significant difference in percentage. For both national and provincial marine ranches, more than $60 \%$ of enterprises are not in the security grade, and they need to improve their ecological security performance. Second, the average MRES values of the national and provincial marine ranches are in the medium security grade and are very close. This means that there is no significant difference between the investigated national and provincial marine ranches in average MRES values; the provincial average MRES value (0.62) is slightly higher than the national (0.61). The above analysis shows that the performance of national marine ranches in ecological security is not necessarily higher than that of provincial marine ranching. The ecological security evaluation value of marine ranching is not related to its rating level. 

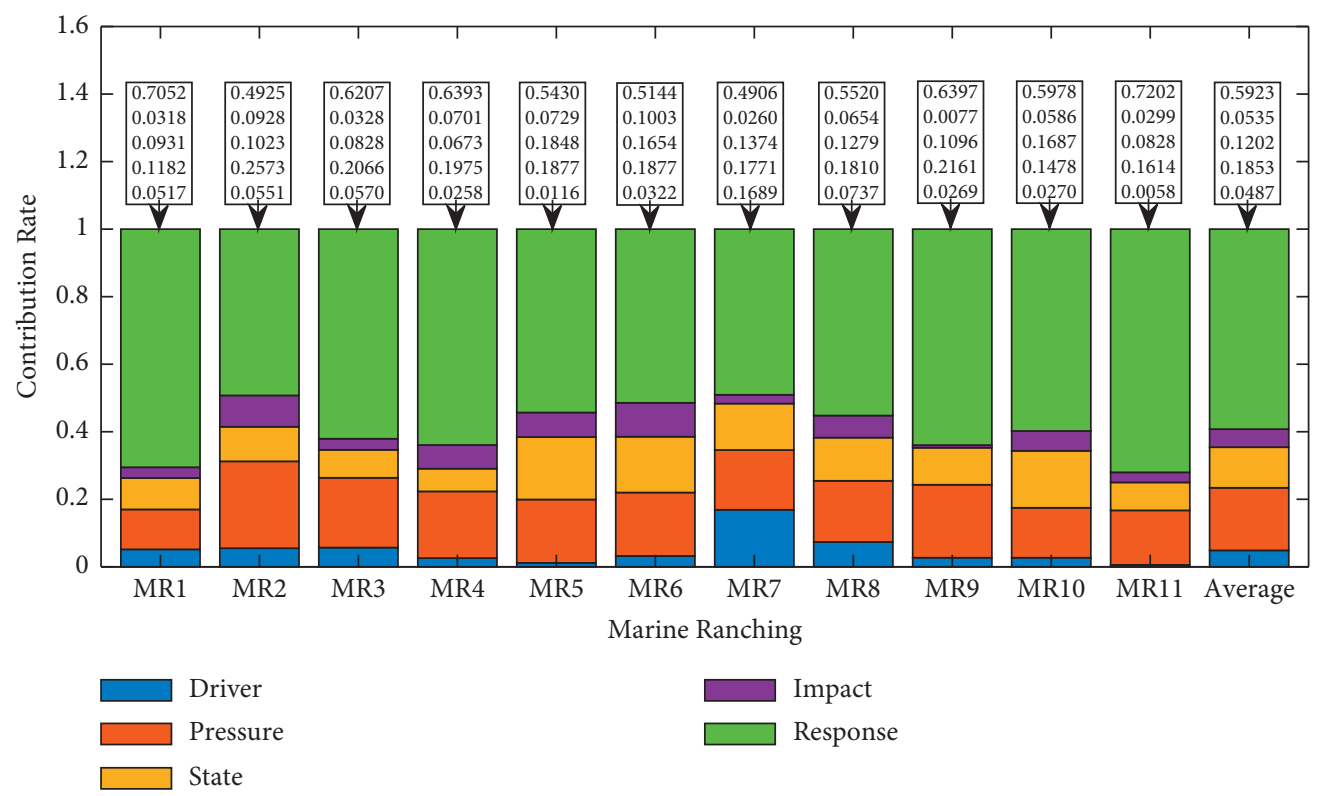

Figure 6: Contribution rates of clusters for MRES.

3.2.3. Contribution Rate Analysis. As mentioned in Section 2.5 , the contribution rate is calculated by equation (17); it reflects the extent to which each cluster contributes to the MRES for each marine ranching. Then, the average contribution rate of each cluster, which reflects the average cluster contribution rate performance for the marine ranches, can be calculated accordingly. The calculation results are shown in Figure 6, where the data of each marine ranching from top to bottom represent the contribution rates of response, impact, state, pressure, driver.

As shown in Figure 6, the contribution rates of the five clusters to MRES in Rongcheng are different. For both national and provincial marine ranching, the contribution rate of response is significantly higher than other clusters, and the contribution rates of driver and impact are generally lower than others. The average contribution rate also reflects this feature; we can see that response is the most important cluster for MRES value with 59\% contribution rate; the average contribution rates of pressure and state are $19 \%$ and $12 \%$, and the lowest average contribution rates are driver and impact with $5 \%$. This indicates that all the investigated marine ranches of Rongcheng are mainly driven by the response cluster. In addition, there is no distinction in national and provincial marine ranching because both of them show consistency in Figure 6. To find the underlying problems of the marine ranches of Rongcheng, we conducted an in-depth analysis of each cluster.

For the response cluster, $\mathrm{MR}_{11}$ has the highest response contribution rate with $72 \%$, and $\mathrm{MR}_{2}$ and $\mathrm{MR}_{7}$ have the lowest response contribution rates with $49 \%$. As the highest contribution rate cluster of MRES, the response cluster is the important subsystem reflecting some positive actions of marine ranches in Rongcheng. For example, nine of 11 marine ranches have constructed "visualization, intelligence, information system;" seven have fixed marine management expenditures; and all follow scientific and sustainable marine halieutics. In addition, marine ranches have positive achievements in talent introduction, scientific cooperation, biotechnology application, and annual monitoring. In documents available for review, the government of Rongcheng established a favorable environment in terms of marine fisheries regulation and science and technology support. These demonstrate that marine ranches and the government attach great importance to science and technology construction, marine ranching's management and maintenance, and monitoring and reporting. In sum, the response is the main driving cluster of MRES in Rongcheng marine ranches, and enterprises and the government have the best performance in the response cluster.

For the driver and impact clusters with an average contribution rate of $5 \%$, the respective maximum values for contribution rates are $17 \%$ and $9 \%$ of $\mathrm{MR}_{7}$ and $\mathrm{MR}_{2}$, and the corresponding minimum values for contribution rates are $1 \%$ and $1 \%$ of $\mathrm{MR}_{11}\left(\mathrm{MR}_{5}\right)$ and $\mathrm{MR}_{9}$. As the two clusters with the lowest contribution to MRES, driver and impact have a small influence on the MRES in Rongcheng. For example, even though a series of measures are taken by the government and marine ranching in ecological environment improvement and resource protection, the performance of marine ranching on the index "enterprise's ecological awareness" is not consistent with this. In the sample period, the proportion of environmental protection expenditure is 0 for $36 \%$ marine ranches; the proportion of environmental protection expenditure is $1 \%$ for $46 \%$ marine ranches; and the proportion is $2 \%$ for $18 \%$ marine ranches. There is no obvious improvement of fishery resources, which indicates the improvement of biodiversity index, and target biomass is not successful. Moreover, as the calculated cluster weights of driver and impact are 0.22 and 0.23 in Section 3.2.1, this indicates that paying attention to these two clusters is necessary for pursuing MRES in experts' views. In sum, although driver and impact are not the main driving clusters 
for MRES in Rongcheng, we can improve overall MRES value to a higher level by protecting resources and establishing awareness of ecological environment protection.

For the pressure and state clusters with average contribution rates of $12 \%$ and $19 \%$, although these two clusters are not the main driving indexes of MRES, they are significant in terms of the MRES. The contribution rates of pressure and state are between the lowest and the highest, which is determined by the specific performance of marine ranching in these clusters. For example, each marine ranching in the sample has put net cages by green framing methods and carried out the construction of artificial reefs, which have had a positive influence. However, in some indexes, the performance of different marine ranching may be very different, or there is room for further improvement. For example, $45 \%$ of the marine ranches scored zero on the index "seaweed field and seagrass bed transplant cultivation," while other marine ranches performed better in terms of the survival status of seaweed field and seagrass bed transplant cultivation. A total of $73 \%$ of the marine ranches reached the national level II standard of seawater quality, and $27 \%$ of the marine ranches reached the highest level I standard. In sum, the contribution of pressure and state to MRES is limited. Marine ranching performs well in artificial reef construction and maintenance, but there is room for further improvement in the marine environment and the construction of seaweed field and seagrass bed.

\subsection{Discussion and Recommendations}

3.3.1. Discussion. First, the study offers the prioritization of evaluation indexes determined by the PLTS/ANP method. "Scientific management of fishery resources" is the most important evaluation index among all indexes. A possible explanation is that scientific management of fishery resources is a systematic project in the MRES construction, including not only the scientific management of marine fishery organisms but also the scientific management of marine ranch staff. The scientific management of fishery resources can be realized mainly through the following three ways. The ratio of feed to breeding objects should be within a reasonable range. In fishing production, compliant fishing methods and fishing tools should be used to selectively capture fishery organisms. They should set up the harvesting range of value-added organisms to achieve sustainable utilization. This shows that the scientific management of fishery resources involves a wide range of measures, which may lead to this index being the most important index in the weight calculation dominated by expert judgment.

The response cluster has the first priority among other clusters, which shows that the response cluster and all its indexes have more effects on MRES. A possible explanation is that the response cluster is the only cluster that has relationships with the other four clusters in the DPSIR model. This in itself gives important meaning to the response cluster. The indexes under the response cluster have an internal influence on the four clusters of driver, pressure, state, and impact. This relationship is ultimately reflected in the priority weight of MRES; that is, the weight of response is the largest. Furthermore, as the cluster that reflects the positive measures taken by enterprises to improve the MRES performance, the response includes indexes that reflect the following three points: the level of information construction and human resources construction of marine ranching, the scientific nature and input intensity in fishery resources management of marine ranching, and the development of annual monitoring activities of marine ranching. All these indexes directly reflect the sustainability of marine ranching in the construction of ecological security and can effectively realize the function of marine ranching in environmental protection, resource conservation, and sustainable fishery output.

Second, the comprehensive evaluation value of MRES offers evidence supporting that the current marine ranches in Rongcheng are at the medium security grade on the whole. For all the marine ranches in the sample, only $38 \%$ are at the security grade and can be recognized as performing well in ecological security. The overall situation is at an acceptable level, but there is still great room for improvement in the construction of ecological security. A possible explanation is that Rongcheng has launched some policies and measures with the government playing the leading role and enterprises serving as the main body in the construction of marine ranching in recent years, such as advocating scientific fishing, monitoring the seawater quality, and strengthening the supervision of marine ranching, which have promoted threshold quality of MRES performance in Rongcheng. At the same time, building a good MRES is not only led by the government. Just as in the case of Rongcheng, there are differences between marine ranches in terms of ecological security; therefore, marine ranches need to further improve the performance of MRES on their own.

Moreover, the evaluation results also suggest that there is not a strong correlation between MRES performance and whether the research objects are national or provincial marine ranching. In fact, there is little difference in the performance of average MRES value and safety level distribution between the eight national marine ranches and three provincial marine ranches in this sample. A possible explanation is that as mentioned above, the government of Rongcheng has made great efforts in policy supervision, marine environmental protection, and other public services, which may make the national and provincial marine ranching consistent in the acquisition of this part of public resources, so there is no difference in the average MRES value and safety level distribution between the MRES of provincial and national enterprises. However, there are only three provincial marine ranches selected in this study, which may lead to poor representability of the data. The average MRES value and safety level distribution calculated may not really represent the ecological security situation of provincial marine ranching in Rongcheng.

Third, the contribution rates of the five clusters to MRES show that response is the most important cluster for MRES in Rongcheng. Response's 59\% average contribution rate indicates that all marine ranches of Rongcheng in this sample are mainly driven by the 
response cluster. A possible explanation is that Rongcheng attaches great importance to information construction, scientific research support, and talent introduction. For example, the average value of cooperation between marine ranches and universities is 2.5, and $82 \%$ of the marine ranches have built visualization, intelligence, and information systems. It can be considered that for marine ranches in Rongcheng, the impact of the response cluster of the DPSIR evaluation model is the highest, and the evaluation indexes under the cluster have great importance. Therefore, the response cluster is an important source of strong MRES performance. From another point of view, while ensuring response cluster performance, we can further enhance MRES performance through other clusters with low contribution rates but high priority weight as calculated by the PLTS/ANP method. For example, although driver and impact clusters have the lowest contribution rates, we can enhance MRES by protecting resources and establishing awareness of ecological environment protection.

3.3.2. Recommendations. MRES, as a hot spot in the research of marine ranching, is of great significance for marine ecological security and the construction of marine ranching. The overall MRES performance in Rongcheng is at a medium level, and there is room for further enhancement. Based on the research results and analysis of MRES evaluation index weights and overall MRES values of marine ranches in this study, the following four suggestions are put forward to help enterprises, government, and third-party institutions work together to improve the ecological security level of marine ranches in Rongcheng.

First, according to the MRES evaluation index system constructed in this study and the weight results, marine ranching should pay attention to the scientific management of fishery resources and consciously improve enterprises' awareness of ecological and environmental protection. Furthermore, for enterprises, it is necessary to emphasize profitability and to pursue profit margins. These three aspects represent the first three important indexes in the evaluation index system. In addition, the significance of the response cluster leads enterprises to pay attention to scientific and technological capabilities, talent introduction, and marine ranching monitoring, among others.

Second, the construction of MRES is inseparable from the government's policy and supervision. The government should make clear the important role of the management and guidance of MRES, establish, and improve the supervision, evaluation, and management mechanism for MRES construction. Specifically, this includes strengthening the improvement and protection of seawater quality, ensuring the scientific fishing of marine living resources, and introducing support policies for MRES. These are the reasons why some marine ranches perform well or perform poorly in the previous analysis.

Third, marine ranches in Rongcheng are driven by the response cluster. In this context, enterprises can further enhance their performance in response to improve the
MRES level. However, the clusters with low contribution rates but not low priority weights like driver or impact cluster should not be ignored to improve the MRES level. To improve the performance of marine ranching in these clusters, which are not the main driving forces for MRES, it will be more challenging for enterprises. However, it is necessary for improving the MRES level.

Fourth, the participation of third-party stakeholders should be considered in the construction of MRES. Talent and technology support are of great significance for MRES, and most of them are related to the indexes in the response cluster. Therefore, marine ranching should establish cooperative relationships of knowledge sharing and joint achievements with scientific research institutes and universities and establish directional training relationships for talent. The government can also guide the collaboration between marine ranches and third-party institutions to transfer technology and knowledge to enterprises and encourage innovative academic research related to MRES.

\section{Conclusion}

In recent years, the research on marine ranching has made rich achievements in concepts, ecological evaluation, breeding carrying capacity, resources, and environmental monitoring. The ecological benefits of environmental protection and resource conservation of marine ranching have been highlighted by the government and academia, but there are still many deficiencies in the practical operation and theoretical research. Therefore, it is necessary to study the evaluation of MRES and to measure the performance of system security and ecosystem services of marine ranching. This study evaluates MRES based on the DPSIR model and PLTS/ANP method and performs a case study of 11 marine ranches in the city of Rongcheng. The main contributions of this study can be summarized as follows.

First, the network model of MRES evaluation constructed by the ANP method reflected the interdependent relationships of evaluation indexes. In the existing research on MRES, the interdependent relationships between indexes are seldom considered, while the relationship between indexes is mostly treated as a linear one. However, considering that the marine ranching ecosystem is a subsystem of the marine ecosystem, it is necessary to reflect the internal and external dependence of the evaluation indexes. Second, the weights of indexes were determined by the PLTS/ANP method. PLTS is practical for expressing experts' preferences and hesitant views, and this method reflects the probability information of each language term. We used PLTS to provide not only the linguistic values but also the corresponding probabilistic information in obtaining the pairwise matrices. Third, the proposed MRES evaluation index system and PLTS/ANP method were applied to analyze 11 marine ranches in Rongcheng, Shandong Province. Four of them were in the insecurity grade, three in the medium security grade, and four in the security grade. Moreover, according to the analysis of index weights, comprehensive MRES evaluation values, and contribution rates of 11 marine ranches, the recommendations for government, enterprises, 
and third-party institutions were proposed to improve MRES performance.

However, there are limitations to this study. First, in this study, PLTS and ANP, which were used to determine the weights, are two subjective methods. Further research can integrate objective methods such as the entropy weight method to obtain the weights for simultaneous subjective and objective analysis. Second, the method for order relations and operations is recently introduced on the set of PLTS, and further research may be conducted with this new method to improve the effectiveness of MRES evaluation results.

\section{Data Availability}

The data used to support the findings of this study are currently under embargo while the research findings are commercialized. Requests for data, 12 months after publication of this article, will be considered by the corresponding author.

\section{Conflicts of Interest}

The authors declare that they have no conflicts of interest.

\section{Acknowledgments}

This research was supported by the Major Program of the National Social Science Foundation of China under grant no. 18ZDA055.

\section{References}

[1] H. S. Yang, S. Y. Zhang, and X. M. Zhang, "Strategic thinking on the construction of modern marine ranching in China," Journal of Fisheries of China, vol. 43, pp. 1255-1262, 2019.

[2] C. K. Zeng, "Some problems on fisheries and animal husbandry production in China's exclusive economic area," Natural Resources, vol. 3, no. 1, pp. 58-64, 1979.

[3] P. M. Chen, L. M. Shu, and H. R. Yuan, "Review on development, definition and classification of marine ranching in domestic and overseas," Journal of Fisheries of China, vol. 43, pp. 1851-1869, 2019.

[4] X. Zhou, X. Zhao, S. Zhang, and J. Lin, "Marine ranching construction and management in east China sea: programs for sustainable fishery and aquaculture," Water, vol. 11, no. 6, p. 1237, 2019.

[5] Z. Y. Li, Q. Lin, J. Li, and X. Shan, "Present situation and future development of marine ranching construction in China," Journal of Fisheries of China, vol. 43, pp. 1870-1880, 2019.

[6] Y.-W. Du and X.-L. Sun, "Influence paths of marine ranching ecological security in China based on probabilistic linguistic term sets and qualitative comparative analysis," International Journal of Fuzzy Systems, vol. 23, no. 1, pp. 228-242, 2021.

[7] Y. W. Du and W. M. Cao, "Theoretical framework for the supervision of the ecological security of China's marine ranching, China. popul. resour," Environ, vol. 31, pp. 182-191, 2020.

[8] Y. W. Du and K. Gao, "Ecological security evaluation of marine ranching with AHP-entropy-based TOPSIS: a case study of Yantai, China," Marine Policy, vol. 122, Article ID 104233, 2020.

[9] M. Qin, X. Wang, Y. Du, and X. Wan, "Influencing factors of spatial variation of national marine ranching in China," Ocean \& Coastal Management, vol. 199, Article ID 105407, 2021.

[10] X. Wan, X. Liu, Z. Du, and Y. Du, "A novel model used for assessing supply chain sustainability integrating the ANP and ER approaches and its application in marine ranching," Journal of Cleaner Production, vol. 279, Article ID 123500, 2021.

[11] Y.-W. Du and X.-X. Li, "Hierarchical DEMATEL method for complex systems," Expert Systems with Applications, vol. 167, Article ID 113871, 2021.

[12] Y.-W. Du and J.-J. Zhong, "Generalized combination rule for evidential reasoning approach and Dempster-Shafer theory of evidence," Information Sciences, vol. 547, pp. 1201-1232, 2021.

[13] T. Ding and L. Liu, "Evaluation of performance of intellectual property strategy based on fuzzy analytic hierarchy process - - taking promotion plan for implementation of intellectual property strategy in jiangsu Province as an example," J. UESTC (Social Sciences Edition), vol. 17, pp. 60-63, 2015.

[14] Y. Teng, X. S. He, and D. B. Ni, "Risk assessment of private equity investment project based on analytic hierarchy process and fuzzy comprehensive evaluation: a case study on 3 private equity investment projects," J. UESTC (Social Sciences Edition), vol. 19, pp. 67-73, 2017.

[15] T. L. Saaty, Decision Making with Dependence and Feedback: The Analytic Network Process: the Organization and Prioritization of Complexity, RWS publications, Pittsburgh, Pennsylvania, 1996.

[16] T. L. Saaty, "Fundamentals of the analytic network process dependence and feedback in decision-making with a single network," Journal of Systems Science and Systems Engineering, vol. 13, no. 2, pp. 129-157, 2004.

[17] T. L. Saaty and H. J. Zoffer, "Negotiating the Israeli-palestinian controversy from a new perspective," International Journal of Information Technology and Decision Making, vol. 10, no. 01, pp. 5-64, 2011.

[18] Y.-T. Lin, C.-L. Lin, H.-C. Yu, and G.-H. Tzeng, “A novel hybrid MCDM approach for outsourcing vendor selection: a case study for a semiconductor company in Taiwan," Expert Systems with Applications, vol. 37, no. 7, pp. 4796-4804, 2010.

[19] R. Yu and G.-H. Tzeng, "A soft computing method for multicriteria decision making with dependence and feedback," Applied Mathematics and Computation, vol. 180, no. 1, pp. 63-75, 2006.

[20] T. Liu, Y. Deng, and F. Chan, "Evidential supplier selection based on DEMATEL and game theory," International Journal of Fuzzy Systems, vol. 20, no. 4, pp. 1321-1333, 2018.

[21] Y. Wu, J. Wang, S. Ji, and Z. Song, "Renewable energy investment risk assessment for nations along China's Belt \& Road Initiative: an ANP-cloud model method," Energy, vol. 190, Article ID 116381, 2020.

[22] M. Roy, P. Sen, and P. Pal, “An integrated green management model to improve environmental performance of textile industry towards sustainability," Journal of Cleaner Production, vol. 271, Article ID 122656, 2020.

[23] Y. Wu, B. Zhang, C. Xu, and L. Li, "Site selection decision framework using fuzzy ANP-VIKOR for large commercial rooftop PV system based on sustainability perspective," Sustainable Cities and Society, vol. 40, pp. 454-470, 2018.

[24] R. M. Rodriguez, L. Martinez, and F. Herrera, "Hesitant fuzzy linguistic term sets for decision making," IEEE Transactions on Fuzzy Systems, vol. 20, no. 1, pp. 109-119, 2012. 
[25] V. Torra and N. Yasuo, "On hesitant fuzzy sets and decision," in Proceedings of the 2009 IEEE International Conference on Fuzzy Systems, pp. 1378-1382, IEEE, Jeju, Korea, August 2009.

[26] L. A. Zadeh, "The concept of a linguistic variable and its application to approximate reasoning-III," Information Sciences, vol. 9, no. 1, pp. 43-80, 1975.

[27] C. Wei, N. Zhao, and X. Tang, "Operators and comparisons of hesitant fuzzy linguistic term sets," IEEE Transactions on Fuzzy Systems, vol. 22, no. 3, pp. 575-585, 2014.

[28] X. Chen, L. Peng, Z. Wu, and W. Pedrycz, "Controlling the worst consistency index for hesitant fuzzy linguistic preference relations in consensus optimization models," Computers \& Industrial Engineering, vol. 143, Article ID 106423, 2020.

[29] P. Wang, P. Liu, and F. Chiclana, "Multi-stage consistency optimization algorithm for decision making with incomplete probabilistic linguistic preference relation," Information Sciences, vol. 556, pp. 361-388, 2021.

[30] Z.-p. Tian, R.-x. Nie, and J.-q. Wang, "Consistency and consensus improvement models driven by a personalized normalization method with probabilistic linguistic preference relations," Information Fusion, vol. 69, pp. 156-176, 2021.

[31] X. Wu and H. Liao, "An approach to quality function deployment based on probabilistic linguistic term sets and ORESTE method for multi-expert multi-criteria decision making," Information Fusion, vol. 43, pp. 13-26, 2018.

[32] C. Zhang, H. Zhang, and J. Wang, "Personalized restaurant recommendation method combining group correlations and customer preferences," Information Sciences, vol. 454-455, pp. 128-143, 2018.

[33] C. Song, X. K. Wang, and P. F. Cheng, "SACPC: a framework based on probabilistic linguistic terms for short text sentiment analysis," Knowledge-Based Systems, vol. 194, Article ID 100572, 2020.

[34] C. Bai, R. Zhang, L. Qian, and Y. Wu, "Comparisons of probabilistic linguistic term sets for multi-criteria decision making," Knowledge-Based Systems, vol. 119, pp. 284-291, 2017.

[35] X. Zhou, L. Wang, J. Qin, J. Chai, and C. Q. Gómez Muñoz, "Emergency rescue planning under probabilistic linguistic information: an integrated FTA-ANP method," International Journal of Disaster Risk Reduction, vol. 37, Article ID 101170, 2019.

[36] B. Li, Z. Xu, and Y. Zhang, "A probabilistic linguistic evaluation-based multi-stage medical scheme selection process related to referral system," Expert Systems with Applications, vol. 170, Article ID 114523, 2021.

[37] OECD, "OECD Environmental Directorate Monographs," OECD, Paris, 1993.

[38] United Nations Commission on Sustainable Development, Indicators of Sustainable Development: Framework and Methodologies, United Nations, New York, NY, USA, 1996.

[39] EEA, The EEA and its Role in Encouraging Better Water Management, EWPCA workshop on environmental technologies, Copenhagen, 1996.

[40] Z. Boaz and B. Assaf, "Ranching fish using acoustic conditioning: has it reached a dead end?" Aquaculture, vol. 344-349, pp. 3-11, 2012.

[41] H. Huang, B. Chen, and Z. Y. Ma, "Assessing the ecological security of the estuary in view of the ecological services- A case study of the Xiamen Estuary," Ocean \& Coastal Management, vol. 137, pp. 12-23, 2017.

[42] B. Sun, J. Tang, D. Yu, Z. Song, and P. Wang, "Ecosystem health assessment: a PSR analysis combining AHP and FCE methods for Jiaozhou Bay, China1," Ocean \& Coastal Management, vol. 168, pp. 41-50, 2019.

[43] W. Lu, C. Xu, J. Wu, and S. Cheng, "Ecological effect assessment based on the DPSIR model of a polluted urban river during restoration: a case study of the Nanfei River, China," Ecological Indicators, vol. 96, pp. 146-152, 2019.

[44] Z. Wang and P. Zhu, "Multi-attribute group three-way decision making with degree-based linguistic term sets," International Journal of Approximate Reasoning, vol. 137, pp. 69-93, 2021.

[45] Z. Zhang and C. Guo, "Fusion of heterogeneous incomplete hesitant preference relations in group decision making," International Journal of Computational Intelligence Systems, vol. 9, no. 2, pp. 245-262, 2016.

[46] B. Marta and M. Giulio, "An appraisal of analytic network process and its role in sustainability assessment in Northern Italy," Manag. Environ. Qual. :Anais: An International Journal, vol. 19, pp. 642-660, 2008.

[47] M. Giannakis, R. Dubey, I. Vlachos, and Y. Ju, "Supplier sustainability performance evaluation using the analytic network process," Journal of Cleaner Production, vol. 247, Article ID 119439, 2020.

[48] Y.-W. Du, B.-Y. Li, and X.-J. Quan, "Construction and application of DPPD model for evaluating marine resources and environment carrying capacity in China," Journal of Cleaner Production, vol. 252, Article ID 119655, 2020. 